\title{
Organisationsentwicklung mithilfe von Wertenetzen und subjekt-orientiertem Geschäftsprozessmanagement
}

\author{
Christian Stary 1
}

Eingegangen: 17. März 2018 / Angenommen: 24. Mai 2018 / Online publiziert: 31. Mai 2018

(C) Der/die Autor(en) 2018

Zusammenfassung Organisationsentwicklung vernetzter Handlungsträger bzw. Organisationen auf der Basis von Werten wird zwar stark propagiert, aber methodisch kaum soweit konkretisiert, dass Geschäftsprozesse unmittelbar ableitbar oder ausführbar werden. Dort setzt dieser Beitrag an. Es wird ein Ansatz gezeigt, Arbeitsprozesse mithilfe der Bewertung formalisierter und informeller Austauschbeziehungen strukturiert und nachvollziehbar zu gestalten. Dabei werden die Austauschbeziehungen zunächst in Wertenetzen dargestellt. Die darauf aufbauende Beziehungsanalyse zeigt Interaktionsmuster zwischen Handelnden, die für Arbeitsabläufe bedeutsam sind, auf und legt Quellen sowie Senken von Leistungsflüssen offen. Die erhobenen Muster bilden den Ausgangspunkt für die input-orientierte Wirkanalyse und die output-orientierte Analyse zur Wertschaffung. Letztere dient auch dem Erschließen von Gestaltungs- bzw. Verbesserungspotenzial mit der Ausrichtung auf die Handelnden. Dieses Potenzial wird an Interaktionsbeziehungen sichtbar. Die Ergebnisse der Analysen können unmittelbar in subjektorientierte Geschäftsprozessmodelle überführt werden. Deren Vorteil gegenüber bestehenden Ansätzen liegt in der unmittelbaren Ausführbarkeit und damit Erfahrbarkeit aus der Sicht der jeweils beteiligten Stakeholder. Somit unterstützen derartig kombinierte Ansätze Aufgabenträger ebenso wie Verantwortliche bei der Entwicklung von Organisationen, die sich auf wertebasierte Austauschbeziehungen gründet und auf aktive Teilnahme ausgerichtet ist.

Schlüsselwörter Value Management · Partizipative Organisationsgestaltung · Netzwerkanalyse · Kommunikationsbasiertes Geschäftsprozessmanagement · Subjekt-Orientierung

C. Stary $(\square)$

Institut für Wirtschaftsinformatik-Communications Engineering, JKU Kompetenzzentrum Wissensmanagement, Universität Linz, Altenbergerstraße 69, 4040 Linz, Österreich E-Mail: Christian.Stary@jku.at 


\section{Value Added and Process Management for Networked Actors}

Abstract Although organizational development of networked actors or organizations based on values is widely propagated, it is still a methodical challenge to design executable business processes based on actual value chains. In this paper such an approach is discussed. It structures work processes through assessing formalized and informal exchange relationships between stakeholder roles relevant for a business operation. Exchange relationships are first captured by a specific concept map. The relationship analysis that builds on it reveals interaction patterns between agents that are significant to workflows and exposes sources and sinks of performance flows. The collected maps form the starting point for the input-oriented impact analysis and the output-based analysis for value creation. The latter also triggers development potential for work redesign or improvement while focusing on actor roles. The results of the analyses can be transformed directly to subject-oriented business process models. Their advantage over existing approaches lie in the capability to execute validated behavior specifications for each stakeholder role. Combining the analysis of value chains with business process specification in this way supports both, operators and those responsible for change management, when actively participating in organizational development.

Keywords Value management · Participatory organization design · Network analysis $\cdot$ Communication-based business process management $\cdot$ Subject orientation

\section{Wertschöpfung und Prozessmanagement für vernetzte Handlungsträger}

Vernetze Handlungsträger bestimmen vermehrt das Arbeitsgeschehen (Gebhardt und Häupl 2012). Dabei sind insbesondere wissensintensive Prozesse, welche das Wirtschaftssystem und zunehmend auch andere Gesellschaftssysteme prägen, steter Veränderung unterworfen (vgl. Becker et al. 2012). Diese Systeme sind aufgrund der starken Durchdringung mit IKT (Informations- und Kommunikationstechnologien) sogenannte sozio-technische Systeme. Bei deren Gestaltung steht im Sinne Ropohls (1999) und Zink et al. (2009, S. 236) die wechselseitige Verschränkung von technischen Systemen und Personen im Mittelpunkt. Das Gestaltungsziel spricht sowohl die Unterstützung menschlicher Fähigkeiten als auch die Bedarfserfüllung durch IKT in einem ausgewogenen Verhältnis an - „... to foster the program of shaping both the technical and the social conditions of work, in such a way that efficiency and humanity would not contradict each other any longer. " (Ropohl 1999).

Beteiligte sollten dabei Fähigkeiten und Kompetenzen für arbeitsplatzübergreifende Abläufe erwerben, sodass Arbeitsmittel oder Informationen, die zwischen den Beteiligten ausgetauscht werden, schließlich von allen Beteiligten genutzt werden können (vgl. Baxter und Sommerville 2011). Ein wesentlicher Erfolgsfaktor scheint die Transparenz von Annahmen und damit Wertesystemen der Beteiligten im Rahmen der Gestaltung sozio-technischer Systeme zu sein. Gelingt es, Wertesysteme im Kontext von Arbeitsprozessen für alle Beteiligten durchschaubar aufzubereiten, 
können Unklarheiten effektiver bearbeitet werden (vgl. Grote 2009). Annahmen und wahrgenommene Werte bilden somit einen wesentlichen Kontext der für die Systemgestaltung bedeutsamen funktionalen und kommunikativen Prozesse.

Wertenetze (Allee 2000, 2003, 2008; Stary et al. 2012; Allee und Schwabe 2015) stellen einen etablierten Ansatz dar, Wertesysteme im Rahmen der Gestaltung von Arbeitsorganisation zu berücksichtigen und unter Beteiligung von Handlungsträgern in die Organisationentwicklung einzubringen. Die Abbildung von Änderungsvorschlägen auf Prozessmodelle wurde bislang zwar aus der Sicht von Funktionen, welche im Rahmen der Aufgabenerfüllung zu bewältigen sind, berücksichtigt, nicht aber deren unmittelbare Ausführbarkeit für die Beteiligten. Gelingt es, Prozessmodelle, welche aus den Wertenetz-Analysen hervorgehen, ohne weitere Transformation für die jeweiligen Rollen der Beteiligten auszuführen, wird Arbeitsorganisation interaktiv erfahrbar. Dies hilft bei der Abklärung, ob Prozesse operativ eingeführt werden sollen, oder beispielsweise zunächst unerwünschte Seiteneffekte zu untersuchen sind.

Wir motivieren den Einsatz von Wertenetzen und erläutern ihre Konzeption in Abschn. 2. Danach wird auf die praktische Arbeit mit Wertenetzen und die Umsetzbarkeit von erkanntem Veränderungspotenzial mittels subjekt-orientierten, ausführbaren Prozessmodellen in den Abschn. 3 und 4 eingegangen.

\section{Formalisierte und informelle Austauschbeziehungen}

Die Analyse und Gestaltung von wertebezogenen Austauschbeziehungen mittels Wertenetz-Analysen ist auf Organisationen als allgegenwärtige, vernetzte Systeme ausgerichtet. Die Vernetzung zeigt sich nicht nur in strategischen Allianzen und in den Lieferanten- und Kundenbeziehungen, sondern vor allem in den internen Beziehungen von Rollenträgern. Sie betreffen somit die Arbeitsweise innerhalb von Organisationen (z. B. Communities, Projektteams). Damit gibt es kaum mehr zentralisierte Stellen, eher selbst organisierende Prozessbeteiligte, die es zu verstehen gilt und die als aktiver Teil der Systemgestaltung zu berücksichtigen sind (Allee 2000; Allee und Schwabe 2015).

Da Prozessbeteiligte vermehrt zu viele unterschiedliche Faktoren bei ihrem Arbeitshandeln gleichzeitig zu berücksichtigen haben, ist Management im klassischen Sinne (Planung, Steuerung und Kontrolle) nicht mehr möglich (vgl. Allee 2003). Vielmehr besteht die zentrale Aufgabe des Managements darin, eine neue Kultur der Zusammenarbeit zu fördern und zu formen (Allee und Schwabe 2015). Die entwickelte Value Network Analysis dient dazu, die eigene(n) Rolle(n) verstehen zu lernen, um anhand individueller Vorschläge von Verhaltensänderungen die Organisation zielgerichtet ändern zu können. Obwohl jede handelnde Person letztlich nur sich selbst steuern kann, kann durch geändertes Verhalten handelnder Personen ein Gesamtsystem beeinflusst werden.

Der Ansatz betont praktisch umsetzbare Teile. Dabei wird ein komplexes System nicht nur in seine Teile oder Prozesse zerlegt und für diese eine möglichst hohe Ertragserzielung gestaltet. Eine derartige Vorgehensweise hilft ja kaum der organisationalen Dynamik gerecht zu werden, insbesondere wenn versucht wird, zerlegte 


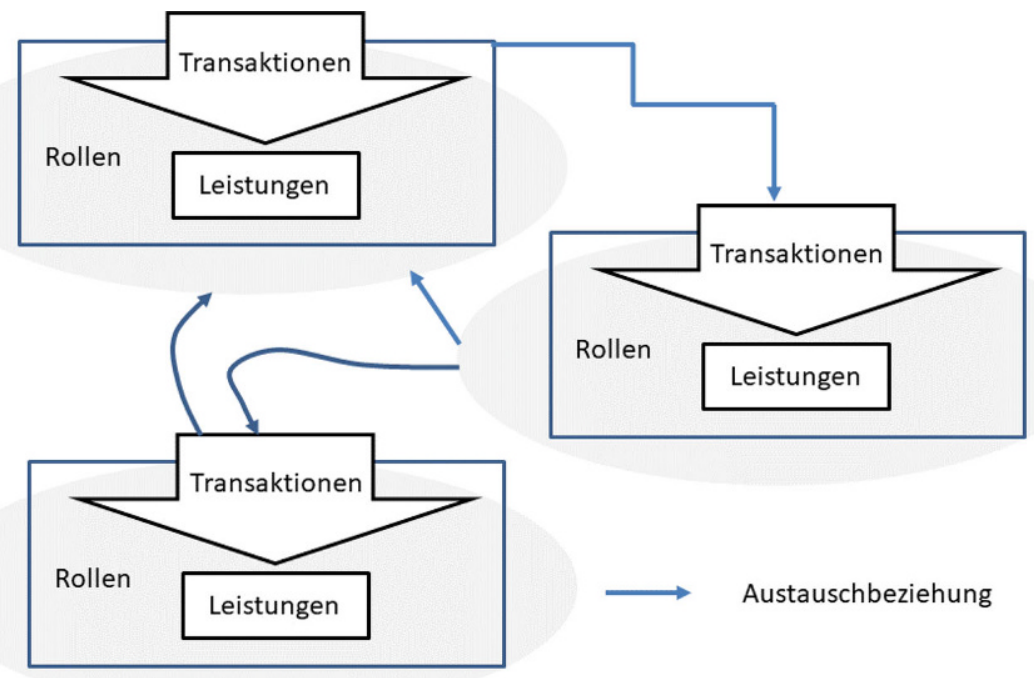

Abb. 1 Konzeption von Wertenetzen nach Allee und Schwabe (2015)

Systeme, deren Teile für sich optimiert wurden, zu einem (optimierten) Gesamtsystem zusammenzusetzen. Grundlegende Organisationsmuster zur Aufgabenbewältigung sind vielmehr formalisierte oder tangible sowie informelle oder intangible Austauschbeziehungen in einem Netzwerk von Akteuren (siehe Abb. 1). Sie stellen die Bausteine wirtschaftlich relevanten Handelns dar.

Nach Allee und Schwabe (2015) werden Austauschbeziehungen über miteinander in Beziehung stehende Rollen festgelegt. Rollenträger erbringen Leistungen, welche über Transaktionen mit anderen Rollen, welche sich im selben oder einem anderen Netzwerk befinden, ausgetauscht werden. Sie bedienen sich im Rahmen der Leistungserstellung dem tangiblen oder intangiblen Vermögensbestand einer Organisation (assets). Zu diesem zählen neben dem finanziellen Vermögen menschliche Kompetenzen, die Marke und bestehende Netzwerkkontakte sowie die interne Struktur, wie sie beispielsweise durch Geschäftsprozesse repräsentiert wird.

Wertschöpfung besteht dabei nicht nur aus formalisierten, d.h. für die Organisation festgelegten Austauschbeziehungen (tangiblen Transaktionen), sondern auch aus darüber hinausreichenden, informellen Austauschbeziehungen (intangiblen Transaktionen). Letztere beziehen sich vor allem auf die Auseinandersetzung über die eigentliche Aufgabenbewältigung hinaus sowie den Austausch derartiger Informationen, da nachhaltiger Erfolg einer Organisation auf Wissensteilung und Offenheit beruht. Beides ermöglicht situationsgerechte Entscheidungsfindung und damit erfolgreiches Bestehen einer Organisation. Wissen und informelle (intangible) Elemente verhalten sich anders als physische Ressourcen im Geschäftsleben, sodass sie nicht als formalisierte (tangible) Elemente betrachtet werden können (Allee 2008). Vielmehr bilden informelle Elemente aufgrund ihrer Nähe zu lebenden Systemen eine eigene Kategorie von Austauschbeziehungen: 
- Formalisierter (Tangibler) Austausch ist definiert über Transaktionen, die Güter, Dienstleistungen oder Erträge beinhalten, z. B. physische Güter, Verträge, Rechnungen, Liefer- und Empfangsbestätigungen, Anfragen, Aufforderungen, Bietereinladungen, Zahlungen. Wesentlich dabei ist, dass wissensintensive Produkte und Dienstleistungen, welche Erträge bewirken und für die Zahlungen als ein Teil einer Dienstleistung oder eines Produkts geleistet werden bzw. aufgrund einer vertraglichen Verpflichtung zu leisten ist, auch als formale Transaktionen betrachtet werden.

- Informeller (Intangibler) Austausch von Wissen und Arbeitsergebnissen unterstützt Kernprozesse und damit die klassische Wertschöpfungskette, unterliegt aber keinerlei vertraglicher Verpflichtung. Informelle Werte sind Aufmerksamkeiten, die Personen einander zukommen lassen, um Beziehungen aufzubauen und Abläufe ohne Störungen bzw. angenehm ablaufen zu lassen. Informelle Transaktionen inkludieren den Austausch strategischer Information, Planungswissen, fachlichoperatives Wissen, gemeinsame Planungsaktivitäten, kollaborative Gestaltung, und bewusstes Vorleben bestimmter Verhaltensmuster. Informelle Transaktionen sind folglich nicht vertraglich vereinbarte Leistungen zum Nutzen oder zur Unterstützung von Organisationen bzw. ihrer Mitglieder. Sie können von einer Person oder Gruppe zu anderen ausgeweitet werden, indem beispielsweise ein Experte für eine bestimmte Zeit von einer Organisationseinheit zur Mitarbeit aufgefordert wird, für den dies Prestige bedeutet. Anerkennung hilft oft in der Beziehungsarbeit, sodass informelle Unterstützung einen wesentlichen Motivationsfaktor für rege Beteiligung und aktive Teilnahme an Gruppenaktivitäten darstellt.

Informelle Austauschbeziehungen stellen nach Allee (2003) das Kernstück allen menschlichen Handelns dar und bestimmen somit auch organisationales und sozio-ökonomisches Handeln. Informelle Transaktionen werden bewusst gesetzt. Sie können herbeigeführt und erkannt werden. Sie sind oft nicht unmittelbar sichtbar, vielmehr ,,verpackt“ in Dienstleistungen oder Produkten. Ein typisches Beispiel ist, Verständnis für eine Kundensituation aufzubauen (intangibel), bevor eine Leistung (tangibel) angeboten wird.

\section{Die Erstellung von Wertenetzen und das Erschließen von Veränderungspotenzial}

$\mathrm{Zu}$ Beginn der Arbeit mit Wertenetzen überlegt jede einzelne teilnehmende Person ihre Rolle, welche sie dann den anderen Teilnehmenden mitteilt. So werden Beziehungen und Wechselwirkungen zwischen den einzelnen Rollen (Personen oder Systemen), die oft nicht bekannt sind, explizit und klarer. Die Rollen werden als Knoten symbolisiert, der Austausch von formalisierten oder informellen wertschöpfenden Leistungen wird in Form von Verbindungen dargestellt, welche die Rollen miteinander verbinden.

Die Modellierung wird bei Wertenetzen Holomapping genannt und bildet die Basis für weitere Analysen. Diese dienen der Wissensauswertung und Wissensverarbeitung und schließen an die Erhebung an. Im Rahmen der Exchange-Analyse 
Abb. 2 Auszug einer Holomap zur umfassenden Klientenbetreuung durch administrative Kräfte im klinikbasierten Gesundheitswesen - tangible Beziehung = durchgängiger Verbindungspfeil, intangible Beziehung $=$ gestrichelter Verbindungspfeil

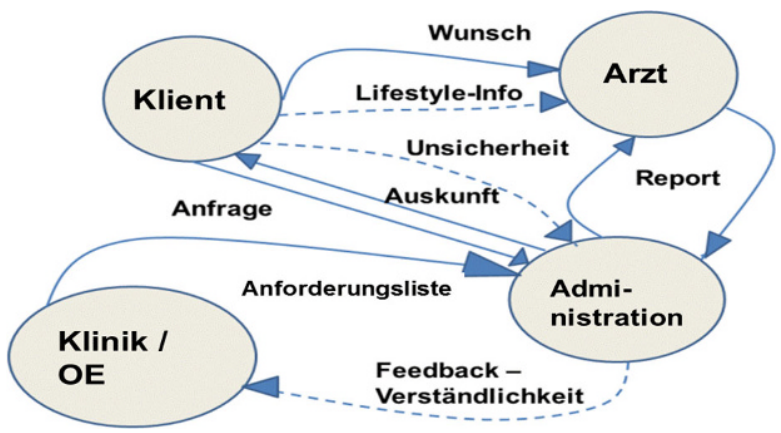

(Austauschanalyse) sowie der Impact-Analyse (Wirkanalyse) und der Value-Creation-Analyse (Analyse zur Werteschaffung) werden die aus der Holomap (dem Wertenetz) sichtbar gewordenen Zusammenhänge in Tabellenform dargestellt und weiter bearbeitet.

Jede einzelne Transaktion steht immer in Beziehung zum gesamten Netzwerk. Wie bereits erwähnt, beziehen sich formalisierte Werteflüsse in der Organisation/im Netzwerk auf den formalisierten Austausch zwischen Personen wie beispielsweise Beispiel Waren, Dienstleistungen und Umsatzerlöse. Sie repräsentieren Transaktionen, die auf Verträgen basieren. Informelle Wertflüsse basieren im Unterschied zu formalisierten Werteflüssen auf Wissen oder einem bestimmten Zusatznutzen. Sie sind nicht vertraglich fixiert oder kostenpflichtig - siehe auch Abb. 2.

Die Austauschanalyse (Exchange-Analyse) untersucht ein Wertenetz auf seine Schlüssigkeit, Robustheit und Nachhaltigkeit. Sie gibt Einblick in die aktuelle Struktur und Dynamik des Netzwerks. Folgende Fragestellungen sollen die ExchangeAnalyse unterstützen:

- Wie fließen wertschöpfende Leistungen durch die Organisation? Zeichnet sich eine bestimmte Logik ab? - Mit Hilfe dieser Fragestellung soll zum einen die Durchgängigkeit der Leistungserbringung thematisiert, und sollen zum anderen Quellen (viele Leistungen an andere) und Senken (kaum Leistung an andere) in der Interaktion sichtbar gemacht werden. Die Durchgängigkeit lässt erwarten, dass Prozesse alle Akteure involvieren, um ein Arbeitsergebnis zu erzielen. Quellen und Senken lassen „Flaschenhälse“ bei Leistungsflüssen erwarten.

- Ist das Verhältnis des Austausches von formalisierten und informellen Leistungen ausgeglichen oder überwiegt eine bestimmte Art des Austausches? - Überwiegt eine Art des Austausches, dann liegt bei überwiegend tangiblen Beziehungen eine stark formalisierte Organisation vor, im gegenteiligen Fall eine stark auf Vertrauen in Leistungen der Akteure ausgerichtete Organisation.

- Ist das Netzwerk optimal durchsetzt oder profitieren bestimmte Teilnehmende auf Kosten anderer? - Dies wird zumeist durch Quellen oder Senken bei den Leistungsflüssen angezeigt.

Die Exchange-Analyse aus Abb. 2 zeigt ausschnittsweise Muster einer Klinik eines Hospitals und damit deren Leistungsbeziehungen: 
- Die Administration ist aus Sicht der Klinik (Organisationseinheit) eine formale Senke - sie empfängt nur Anforderungslisten, sonst werden nur informelle Leistungen (Feedback zur Verständlichkeit der Anforderungen) ausgetauscht.

- Der Arzt bekommt zwar neben einem konkreten Terminwunsch über eine formalisierte Interaktion Lifestyle-Information freiwillig als informelle Information vom Klienten, erbringt aber im Gegenzug keine informellen Leistungen, wie etwa die Übermittlung vertrauensbildender Information.

- Die Übersetzung von Klientenunsicherheit in Richtung Arzt und Klinik lastet auf der Administration.

Eine erste Auswertung dieser Art kann ein Zeichen für die unvollständige Interaktion im Sinne ganzheitlicher Versorgung der Klienten sein. Es existiert zwar eine bestimmte Form der Informationsversorgung. Aber selbst bei einer beidseitig ausgerichteten Interaktion (siehe Administration und Klient), kann es seitens der Klienten gegebenenfalls (tangible) Anfragen geben, die (intangible) Unsicherheit widerspiegeln und Auskunftsleistungen auslösen.

Die Wirkanalyse (Impact-Analyse) untersucht die Auswirkung(en) jedes einzelnen Werteinput auf die Teilnehmenden und legt so ihren Fokus auf die Empfangenden von Werteinputs. Es soll die Frage nach den formalen wie informellen Aufwände, und damit Kosten für interne Strukturaufwände, erforderlicher Kompetenzen und Beziehungsarbeit nach außen, für jeden input aus Sicht der jeweiligen Empfänger beantwortet werden.

Die Impact-Analyse zeigt folglich auf, welcher Input welche Reaktionen und Aktivitäten auslöst und wie sich dieser auf die formalen und informellen Vermögensgegenstände der betroffenen Empfänger auswirkt. Kosten und Nutzen von Werteinputs werden mit niedrig, mittel oder hoch bewertet. Um einen besseren Überblick zu erlangen, werden für alle einzelnen Empfangenden von Werteinputs die Antworten in eine Tabelle eingetragen und die Ist-Situation analysiert.

Tab. 1 zeigt eine Wirkanalyse auf Basis der gewonnenen Erkenntnisse aus der Austauschanalyse der Administration. In der Tabelle wird abgebildet von wem Input für welche Aktivitäten kommt und welche Auswirkungen in Form formalisiert oder informeller Werteflüsse wahrgenommen werden. Wesentlich für die Abschätzung von Veränderungspotenzial sind die Spalteneinträge zu den allgemeinen Kosten und Risiken sowie zum Nutzen von Input.

Die Daten der Auswertung der Austauschanalyse stellen also die Grundlage für die beiden weiteren Analysen dar, wobei aus der Sicht von erhaltenem Input (Wirkanalyse) und übermitteltem Output (Analyse zur Schaffung von Werten) detaillierte Auswertungen von Transaktionen erfolgen. Diese bringen Erkenntnisse für Veränderungsprozesse mit sich. So wird beispielsweise ausgeführt, wann Information erfolgreich übermittelt wird, wie in Tab. 1 ersichtlich. Eine formale Beziehung dieser Art kann helfen, Anfragen zu vermeiden, die Unsicherheit seitens der Klienten widerspiegeln.

Beispielsweise kann im Webauftritt der Klink ein Feature für entsprechenden Informationsaustausch sorgen. Aus den Einträgen der Tabelle zur Liste mit Ressourcenanforderungen (Termine, OP-Saal, Bündelung von Klienten mit bestimmten 


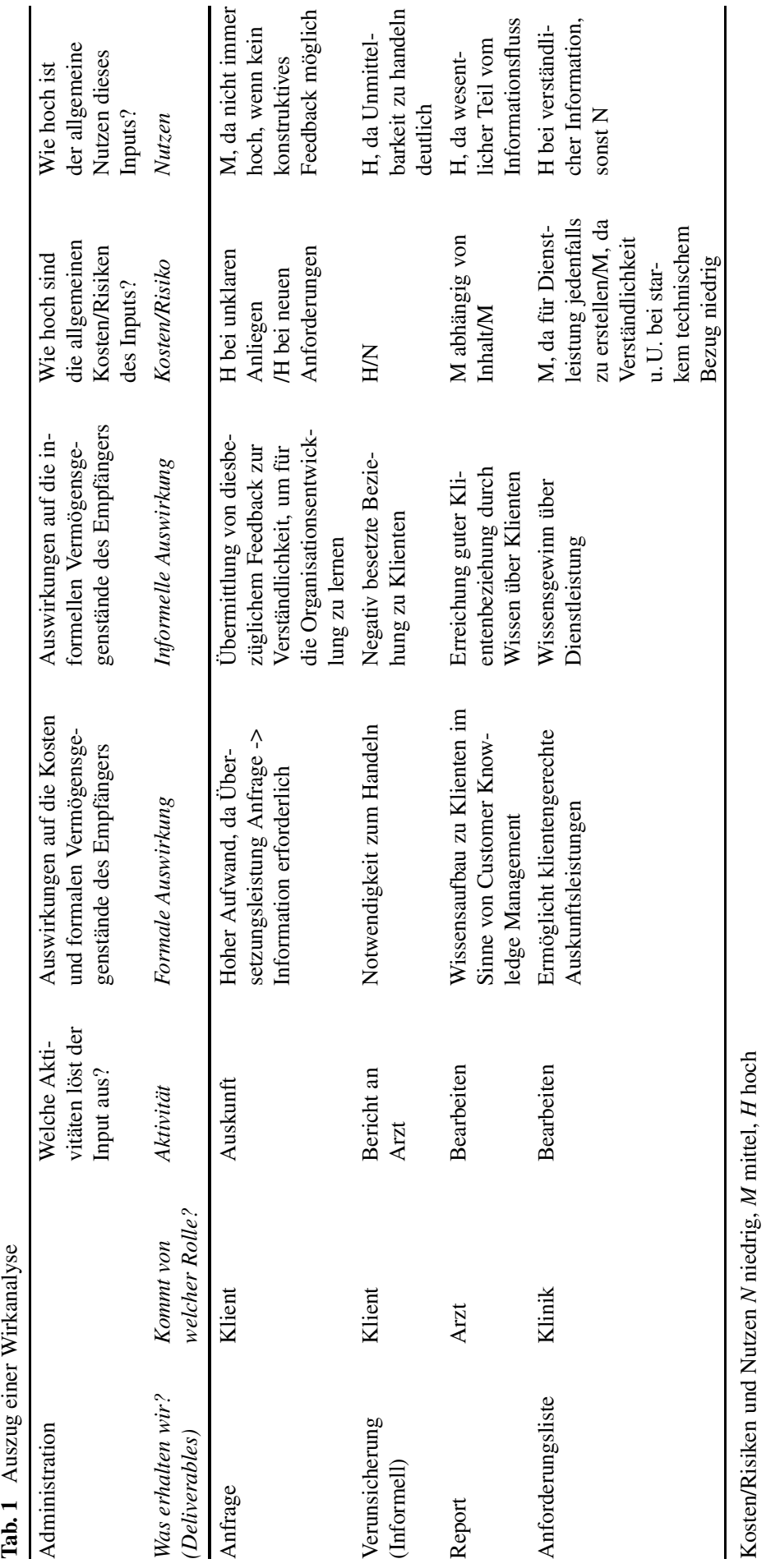


Tab. 2 Auszug aus der fallbezogenen Analyse zur Schaffung von Mehrwert

\begin{tabular}{|c|c|c|c|c|}
\hline Administration & & $\begin{array}{l}\text { Welche Aktivitäten sind beim Sender } \\
\text { mit diesem Output verbunden? Wie } \\
\text { wird zusätzlicher Wert zu diesem } \\
\text { Output geschaffen? }\end{array}$ & & \\
\hline $\begin{array}{l}\text { Output des } \\
\text { Senders }\end{array}$ & $\begin{array}{l}\text { Output } \\
\text { Adressat }\end{array}$ & $\begin{array}{l}\text { Wertsteigerung, Mehrwert der Aktivi- } \\
\text { tät }\end{array}$ & $\begin{array}{l}\text { Kosten/ } \\
\text { Risiken }\end{array}$ & Nutzen \\
\hline $\begin{array}{l}\text { Feedback - } \\
\text { Verständlich- } \\
\text { keit }\end{array}$ & Klinik & $\begin{array}{l}\text { Klientenorientierter Zugang } \\
\text { Berücksichtigung der Machbarkeit }\end{array}$ & $\mathrm{H} / \mathrm{H}$ & $\mathrm{H}$ \\
\hline Auskunft & Klient & $\begin{array}{l}\text { Bedürfnisgerechter Umgang } \\
\text { interessant auch für weitere Klienten }\end{array}$ & $\mathrm{H} / \mathrm{H}$ & $\mathrm{H}$ \\
\hline Report & Arzt & $\begin{array}{l}\text { Gewinnung von Klientendaten } \\
\text { interessant auch für Schulung }\end{array}$ & $\mathrm{H} / \mathrm{H}$ & $\mathrm{H}$ \\
\hline
\end{tabular}

Bei Kosten/Risiken und Nutzen: $N$ niedrig $M$ mittel $H$ hoch

Anliegen etc.) wird ebenfalls der für die Wertschöpfung relevante Bezugspunkt ersichtlich (Qualität der Auskunftsleistung an Klienten durch Administration).

Die Analyse zur Schaffung von Werten (Value-Creation-Analyse) ermittelt, wie Werte bestmöglich erschaffen, erhöht und eingesetzt werden können. Wie die Wirkanalyse betrachtet die Analyse zur Schaffung von Werten auch das Wirken einer bestimmten Rolle im betrachteten Ausschnitt einer Arbeitsorganisation. Im Gegensatz zur Wirkanalyse wird diesmal die output-erzeugende Person in ihrer Rolle und mit ihren diesbezüglichen Aktivitäten betrachtet. Dabei werden alle Werteoutputschaffenden Personen und Handlungen analysiert und auf Basis des ist-Zustands, wie zusätzlicher Mehrwert und Wertezuwachs realisiert werden kann. Hierzu wird eine Kosten/Nutzenabschätzung vorgenommen.

Bei der in Tab. 2 gezeigten Analyse zur Schaffung von Werten wurde anhand erzielter Arbeitsergebnisse analysiert, wie Werte bestmöglich erschaffen, erhöht und eingesetzt werden können. Die Tabelle zeigt die Analyse auf Basis der Daten aus der Austauschanalyse einer Administrationskraft. An Leistungsindikatoren können aus den Analysen die Anforderungen, die an den Arzt sowie die Klinik/ Organisationseinheit gestellt werden, gefiltert werden. Die daraus entwickelte Strategie kann mit der Aussage ,Verfügbarkeit bzw. Transparenz von Information“ umrissen werden.

So kann nun als Veränderungsmaßnahme beschlossen werden, das identifizierte Wertschöpfungspotential im Wertenetz durch Erweiterung der formalisierten Informationsflüsse zwischen allen funktionalen Einheiten zu steigern. Wesentlich zur Entscheidungsunterstützung sind die Einträge in die Spalten „Kosten/Risiken“ und „Nutzen“. Hier kann sich die Einschätzung vor allem bei Kosten und Risiken bei der Soll-Situation relativieren, wenn beispielsweise Inhalte in Schulungen oder Organisationsentwicklungsmaßnahmen aufgenommen werden sollten, da es Know-How zur Erstellung entsprechender Unterlagen sowie der Bereitstellung effektiver Vermittlungsformate im Netzwerk gibt (etwa im Kontext des ohnehin festgelegten Berichtswesens - vgl. „Report“ als dritten Tabelleneintrag in Tab. 2). Eine Soll-Aufstellung berücksichtigt zumeist Wissen über die Machbarkeit bzw. Verfügbarkeit von 
Ressourcen und damit verbundenem Aufwand zur Umsetzung von Maßnahmen, und zwar ohne eine diesbezügliche Entscheidung vorwegzunehmen.

Bislang wurde mit der Durchführung der Austauschanalyse Einblick über die aktuelle Struktur und Dynamik des Netzwerks geschaffen. Die Durchführung der Wirkanalyse erlaubt allen Teilnehmenden ihre eigenen Rollen im Netzwerk im Gesamtzusammenhang zu erschließen. Sie ermöglicht einen Überblick, welche Auswirkungen jede einzelne Wertetransaktion auf die Teilnehmenden hat. Die Analyse zur Schaffung von Werten erlaubt zu bewerten, wie Werte bestmöglich erschaffen, erhöht und eingesetzt werden können und gegebenenfalls auf andere Rollen wirken bzw. diese miteinbeziehen sollten.

Anhand der abgeleiteten Leistungsindikatoren kann eine Strategie entwickelt werden, um das identifizierte Wertschöpfungspotential im Wertenetz zu steigern. Typische Ergebnisse einer Auswertung umfassen fehlende Austauschbeziehungen, also beispielsweise in unserem Fall:

- Konkrete Anfragen an Klienten, um deren Anliegen (insbesondere jene, welche zur Verunsicherung führen) aus Sicht der Klinik und des Arztes besser verstehen zu lernen - die Administration nimmt hier als primäre Anlaufstelle eine Vermittlungsrolle ein, wodurch auch der formale Report aufgewertet werden und das Feedback konkrete Hinweise zur Verbesserung bzw. Schaffung von zusätzlichen Kommunikationsmittel beinhalten kann.

- Feedback von Klienten, das sowohl den zukünftigen als auch den bisherigen Umgang mit Anliegen und damit im Besonderen die Klinik/Organisationseinheit betrifft, etwa Wartezeiten, bis ein Bett verfügbar oder ein Termin zu einer speziellen Behandlung vereinbart wird. Sobald die Verständlichkeit explizit adressiert wird, etwa durch Transparenz von Planungsdaten bezüglich Verfügbarkeit von Personal und Ressourcen, kann ein diesbezüglicher Informationsaustausch mit der Organisationseinheit (und auch dem Arzt) sichergestellt werden. Diese weitere Maßnahme erleichtert den klientengerechten Zugang zur Dienstleistung.

Beide Maßnahmen zeigen die Notwendigkeit vernetzter Darstellung von Austauschbeziehungen. Es ist also nicht unbedingt die Unmittelbarkeit von Austauschbeziehungen, sondern vielmehr die Verkettung von Austauschbeziehungen, wie die Entstehung von Zufriedenheit und Bindung von Klienten, die einen entsprechenden Mehrwert bewirken kann. Würde beispielsweise eine Klinik direkt mit Klienten beginnen zu kommunizieren, wäre zunächst eine bestimmte Gesprächsbasis herzustellen. Derartige Maßnahmen könnten für den zu erreichenden strategischen Zweck sogar kontraproduktiv sein und die bestehende Verunsicherung der Klienten noch erhöhen. Das würde damit dem angestrebten Ziel zuwider laufen.

Die Ergänzungen des Netzwerks erlauben somit eine kontextsensitive Systembetrachtung der Wertschöpfung durch formalisierte und informelle Leistungen, die zwischen den beteiligten Akteuren fließen sollten. Das geänderte Wertenetz (Holomap), wie in Abb. 3 gezeigt, bildet die Grundlage für die weitere Gestaltung. Sie zeigt das Feedback bezüglich Verständlichkeit erstmals als formalisierte Leistung (und ersetzt das bislang informell erbrachte Feedback), wodurch sich die Informiertheit der Beteiligten durch die Transparenz und Verfügbarkeit von Information erhöhen soll. 
Abb. 3 Adaptiertes Wertenetz nach Auswertung der Analyseergebnisse - Legende: tangible Beziehung = durchgängiger Verbindungspfeil, intangible Beziehung $=$ gestrichelter Verbindungspfeil, durchgekreuzter Verbindungspfeil = ersetzte Austauschbeziehung

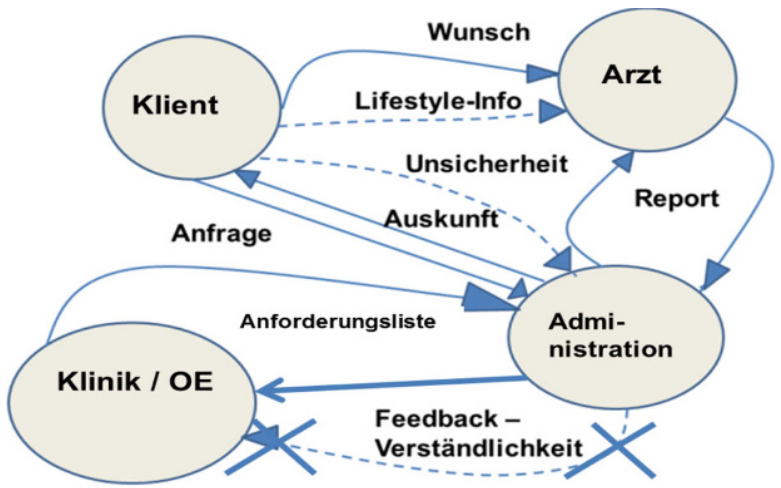

Langfristig anzustreben ist als informeller Austausch zwischen Klienten und Administration ein durch wechselseitige Sicherheit geprägter Umgang. Dieser zeigt sich im Feedback der Klienten und in der Wahrnehmung der Administration, ob die Unsicherheit der Klienten sinkt. Langfristig sollte folglich aus strategischer Sicht das „Unsicherheit“-Wert informeller Art verschwinden und beispielsweise durch ein konstruktives Vorschlagswesen, also formalisierte Austauschbeziehungen ersetzt werden. Die formalisierten Austauschbeziehungen sind es, die den Gestaltungsgegenstand im Geschäftsprozessmanagement darstellen.

\section{Prozesse interaktiv erfahrbar gestalten}

Die interaktive Ausführung von Prozessmodellen unterstützt die unmittelbare Überführung des mittels Wertenetzanalysen geschaffenen Wissens zu jedem Zeitpunkt der Gestaltung oder Umgestaltung formalisierter Austauschbeziehungen. Subjektorientiertes Geschäftsprozessmanagement (S-BPM) (Schmidt et al. 2009; Fleischmann et al. 2011) stellt eine Weiterentwicklung des klassischen Prozessmanagements in Richtung Stakeholder-Orientierung dar. S-BPM erfordert keine eigene Prozessausführungssprache. Vielmehr erlaubt sie mit einem Modell, das Prozessbeteiligte erstellen, die Ausführung und Simulation von Arbeitsabläufen.

Wesentliche Modellbestandteile sind das Subjektinteraktionsdiagramm und das Subjektverhaltensdiagramm. Dazu ein Beispiel aus der Personaleinsatzplanung, welche zur operationalen Umsetzung der oben erwähnten Erhöhung der Transparenz herangezogen werden kann: Mitarbeiter wie Ärzte müssen bei Dienständerungen (Tagung, Urlaub, familiäre Umstände etc.) Dienstrevisionsträge (DRA) stellen, über welche die vorgesetzte Stelle (in der Klinik/Organisationseinheit oder Administration) entscheidet (vgl. Augl 2012). Diese informiert die antragstellende Person über die jeweilige Entscheidung.

Subjekte entsprechen direkt den Knoten der Wertenetzs (Holomaps), also Klient, Arzt, Klinik/Organisationseinheit und Administration. Formalisierte Austauschbeziehungen können ebenfalls direkt von Wertenetzen übernommen werden und werden als Nachrichten entsprechend ihrer Richtung mit Pfeil dargestellt. Für jedes 


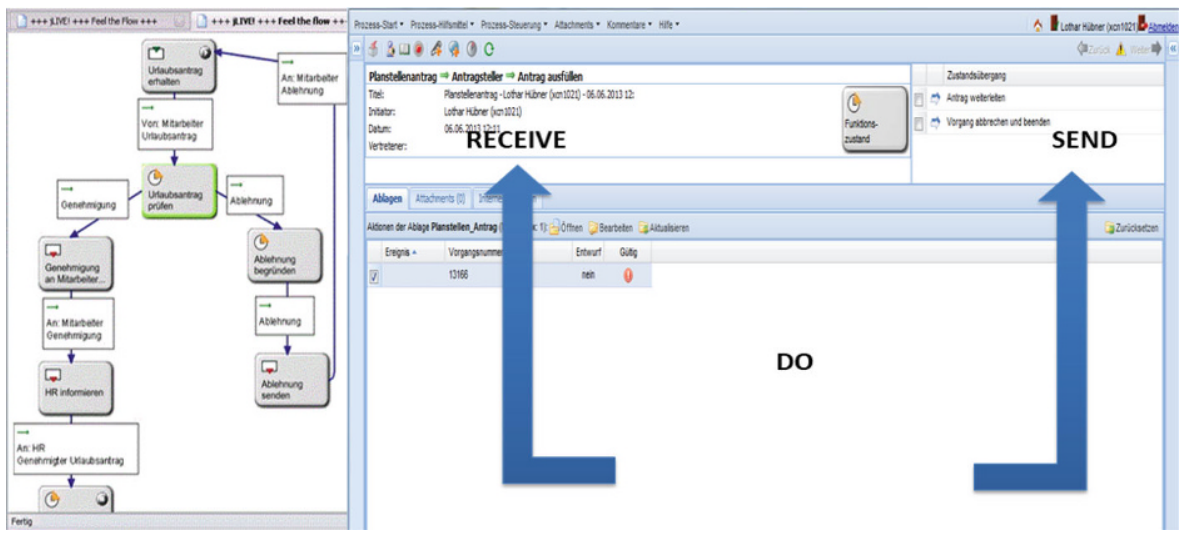

Abb. 4 Duale Sicht - Modell und Ausführung

Subjekt ist sein Verhalten mithilfe dreier Zustände und Übergängen zwischen diesen zu bestimmen: Senden (Sendezustand), Empfangen (Empfangszustand) und Tun (Funktionszustand).

Abb. 4 zeigt einen Ausschnitt bei der Entscheidungsfindung durch den Klinikvorstand bezüglich eines Urlaubsantrags eines Arztes. Das technische System kann dabei vorab prüfen (z. B. durch Zugriff auf eine Funktion des Personalverwaltungsprogramms), ob etwa ein zweiter Arzt im beabsichtigten Urlaubszeitraum auf der Station zur Patientenversorgung verfügbar ist, und dementsprechend einen Vorschlag anbieten. Der Bildschirmbereich ist entsprechend dem Arbeitsfluss in einen Empfangs-, Sende- und Editierbereich aufgeteilt.

Mit Hilfe derartiger Ansätze können Beteiligte (zumeist iterativ) aus ihrer jeweiligen Rolle (mit)gestalten lernen, und zwar unter Berücksichtigung der eigenen Handlungen und im Handlungskontext aller an einem Prozess Beteiligten.

\section{Schlussfolgerung und Ausblick}

Sollen Veränderungen kontinuierlich in Organisationen unter Beteiligung der Handelnden gestaltet werden, stellen deren Modelle sowie eine effiziente methodische und technische Unterstützung wesentliche Ankerpunkte dar. Der vorgestellte Ansatz zur Gestaltung von Arbeit basiert auf Austauschbeziehungen von Werten für die beteiligten Aufgabenträger. Ausgangspunkt stellen individuell wahrgenommene formalisierte (tangible) und informelle (intangible) Austauschbeziehungen dar.

Diese können bestimmte Muster beinhalten, welche mittels der Austauschanalyse transparent gemacht werden. Sie können aus der Sicht eingehender und ausgehender Leistungen von Akteuren (Wirkanalyse und Analyse zur Schaffung von Werten) analysiert werden. Änderungspotential ergibt sich durch das Überdenken von Arbeitsergebnissen (ausgehender Leistungen) mit dem Ziel, für andere Stakeholder Mehrwert zu bewirken. Nehmen sie entsprechende Vorschläge an, können neue Austauschbeziehungen entstehen. 
Zur unmittelbaren Umsetzung der Änderungsvorschläge kann eine kommunikationsorientierte Modellierungstechnik eingesetzt werden, welche auf wenigen, eingängigen Symbolen zur Erstellung von Modellen basiert. Der vorgestellte subjektorientierte Ansatz erlaubt darüber hinaus unmittelbares Ausführen von Modellen, sodass Prozesse mit minimalem Aufwand für Beteiligte (vgl. Freimann und Walther 2012) erfahren werden können. Dies gestattet es Organisationen, ein mit Austausch von Werten begründetes Prozessdesign bereits vor dessen operationaler Einführung zu testen. Die Technikgestaltung rückt zugunsten der Organisationentwicklung in den Mittelpunkt (vgl. Dolata 2011) - ein Potenzial, das es in der Folge gilt, in eine empirisch gesicherte Beleglage zu bringen (vgl. Ackerman et al. 2017).

Funding Open access funding provided by Johannes Kepler University Linz.

Open Access Dieser Artikel wird unter der Creative Commons Namensnennung 4.0 International Lizenz (http://creativecommons.org/licenses/by/4.0/deed.de) veröffentlicht, welche die Nutzung, Vervielfältigung, Bearbeitung, Verbreitung und Wiedergabe in jeglichem Medium und Format erlaubt, sofern Sie den/die ursprünglichen Autor(en) und die Quelle ordnungsgemäß nennen, einen Link zur Creative Commons Lizenz beifügen und angeben, ob Änderungen vorgenommen wurden.

\section{Literatur}

Ackerman M, Goggins S, Herrmann T, Prilla M, Stary C (2017) Designing healthcare that works: a sociotechnical approach. Academic Press, London.

Allee V (2000) Reconfiguring the value network. J Bus Strategy 21(4):36-41

Allee V (2003) The future of knowledge. Increasing prosperity through value networks. Butterworth-Heinemann, Amsterdam

Allee V (2008) Value network analysis and value conversions of tangible and intangible assets. J Intellect Cap 9(1):5-24

Allee V, Schwabe O (2015) Value networks and the true nature of collaboration. Meghan-Kiffer Press, Tampa

Augl M (2012) Building a conceptual roadmap for systemic change - a novel approach to change management in expert organizations in health care. In: Proceedings S-BPM ONE. Lecture notes in business information processing, Bd. 104. Springer, Berlin, S 43-61

Baxter G, Sommerville I (2011) Socio-technical systems: from design methods to systems engineering. Interact Comput 23:144-174

Becker J, Schwaderlapp W, Seidel St (2012) Management kreativitätsintensiver Prozesse. BPM kompetent. Springer, Berlin

Dolata U (2011) Wandel durch Technik: Eine Theorie soziotechnischer Transformation Bd. 73. Campus, Frankfurt

Fleischmann A, Schmidt W, Stary C, Obermeier S, Börger E (2011) Subjektorientiertes Prozessmanagement. Hanser, München

Freimann J, Walther M (2012) Von der Effizienz zur Konsistenz? In: Industrial Ecology Management, S 187-204

Gebhardt B, Häupl F (2012) New work order. bso Verband Büro-, Sitz- und Objektmöbel e. V., Wiesbaden

Grote G (2009) Die Grenzen der Kontrollierbarkeit komplexer Systeme. In: Weyer J, Schulz-Schaeffer I (Hrsg) Management komplexer Systeme. Konzepte für die Bewältigung von Intransparenz, Unsicherheit und Chaos. Oldenbourg, München, S 149-168

Ropohl G (1999) Philosophy of Socio-technical systems, in: society for philosophy and technology 4(3). http://scholar.lib.vt.edu/ejournals/SPT/v4n3/ROPOHL.html. Zugegriffen: 11.11.2017

Schmidt W, Fleischmann A, Gilbert O (2009) Subjektorientiertes Geschäftsprozessmanagement. HMD Prax Wirtschaftsinform 46(266):52-62

Stary C, Maroscher M, Stary E (2012) Wissensmanagement in der Praxis: Methoden-Werkzeuge-Beispiele. Hanser, München

Zink KJ, Kötter W, Longmuß J, Thul MJ (Hrsg) (2009) Veränderungsprozesse erfolgreich gestalten. Springer, Berlin 